Objectives: This study aimed to evaluate the association of serum vitamin A, vitamin $\mathrm{E}$ and folate level with hyperuricemia in the Korean general population. Methods: The present study included 6023 participants (2722 men and 3301 women) aged $\geq 19$ years with available data on serum vitamin $A$, vitamin E, folate and serum uric acid. General characteristics of participants were compared using the Chi-square test and Student's t test. The association between serum vitamin A, E and folate and serum uric acid levels were evaluated using general linear regression model. Multivariate logistic regression analyses were performed to estimate the effects of these micronutrients on hyperuricemia.

Results: Serum uric acid levels were increased from the lowest quintile of vita$\min A$ levels to the highest quintile after adjustment for covariates $\left(P P_{\text {trend }}<0.001\right.$ in both sexes). In addition, dose-dependent relationship was observed between vitamin $A$ levels and the risk of hyperuricemia in fully-adjusted analyses ( $P$ $<0.001$ in both sexes). However, neither serum vitamin $E$ nor serum folate was associated with hyperuricemia across analyses models.

Conclusion: This study suggested that vitamin A could be a risk factor of hyperuricemia and further studies are warranted to elucidate underlying mechanism of the observed findings.

References:

[1] Choi, Woo-Joo, et al. "Independent association of serum retinol and $\beta$ carotene levels with hyperuricemia: A national population study." Arthritis care \& research 64.3 (2012): 389-396.

Disclosure of Interests: : None declared

DOI: 10.1136/annrheumdis-2020-eular.5848

\section{THU0430 RENAL URATE DEPOSITION: SUMMARY OF PUBLISHED EVIDENCE}

P. Khanna ${ }^{1}$, B. Marder ${ }^{2}$, B. Lamoreaux ${ }^{2}$, A. Kumar ${ }^{2} .{ }^{1}$ University of Michigan, Ann Arbor, United States of America; ${ }^{2}$ Horizon Therapeutics, Lake Forest, United States of America

Background: Gout is the most common inflammatory arthropathy in U.S. adults. Although the severity of this debilitating disease is often defined by the presence of tophi in the joints, systemic deposition of urate in major organ systems including the renal parenchyma is not as well established. Urate is primarily cleared through the kidneys and patients with gout often have concomitant renal disease along with other comorbidities such diabetes, coronary artery disease, and hypertension; however, a causal role between these entities has not yet been carefully established. We hypothesize that urate deposits serve as a trigger in the inflammatory nidus to propogate subclinical tissue damage that results in the chronicity of the disease. This could potentially explain its independent role in the development and progression of chronic kidney disease in gout patients.

Objectives: To review the published literature for evidence of urate deposition in the renal parenchyma in patients with gout and summarize the histopathology and imaging findings.

Methods: PubMed (from 1940 to 2020) was used to identify reports of autopsy, pathology and radiology imaging demonstrating urate deposition within the native renal parenchyma in patients with gout. Key words included: gout nephropathy, chronic urate nephropathy, renal tophi, gouty kidney, autopsy findings in gout, and renal imaging in gout. The reference lists from these publications were also used to identify additional articles. Literature referencing urate nephrolithiasis and renal transplants were excluded from the study.

Results: There were 25 articles documenting renal parenchymal urate deposition in gout patients confirmed by autopsy, biopsy and/or radiology imaging in native kidneys. Among the 19 articles examining urate deposition by autopsy and/or biopsy, $100 \%$ found urate deposition in the collecting ducts and adjacent medullary interstitium. Based on these findings, the most commonly proposed mechanism for urate deposition is urate crystal precipitation in the collecting ducts with eventual desquamation of the collecting duct walls from inflammation and/or tubular obstruction with subsequent extrusion of crystals into the medullary interstitium. $89 \%$ of reports documented inflammatory cells and/ or tubulointerstitial fibrosis adjacent to the renal urate deposits. $68 \%$ reported cortical thinning or scarring. In addition, $74 \%$ of included publications reported renal vascular pathology including arteriosclerosis, glomerosclerosis and nephrosclerosis. There were 6 imaging articles that all reported abnormal renal ultrasound findings with hyperechogenic renal medullas that were attributed to urate deposition.

Conclusion: There is a growing body of literature documenting urate deposition in the renal parenchyma in gout patients based on autopsy, pathology and imaging findings. Inflammation and fibrosis adjacent to regions of urate deposition and vascular changes were common. Given the strong association of gout with renal disease, there is a critical need to elucidate the mechanism by which urate impairs the renal tissue. Thus dedicated investigation is key to determine the prevalence and clinical significance of urate deposition in the kidneys of gout patients.

\section{References:}

[1] Nickeleit, V, et al. Nephrol Dial Transplant. (1997) 12:1832-1838.

[2] Tchacarski, V, et al. Intl Urol Nephrol. 1992; 24(6): 649-655.

[3] Modern, FW. Med Clin North Am. 1952;21: 941-51.

[4] Linnane, JW, et al. Nephron. 1981; 29:216-22

[5] Greenbaum, D, et al. Br Med J. 1961 May 27; 1(5238): 1502-1504

[6] Bluestone, R, et al. Seminars in Arthr and Rheum. 1977;7(2).

[7] Brown, J, et al. NEJM. 1950; 243: 325-329.

[8] Braga, T., et al. Sci Rep. 2017; 7: 39884.

Disclosure of Interests: : Puja Khanna Grant/research support from: Dyve, Selecta, Sobi, Consultant of: Sobi, Horizon, Brad Marder Employee of: Horizon Therapeutics, Brian LaMoreaux Shareholder of: Horizon Therapeutics Employee of: Horizon Therapeutics, Ada Kumar Shareholder of: Horizon Therapeutics, Employee of: Horizon Therapeutics

DOI: 10.1136/annrheumdis-2020-eular.1761

\section{THU0431 PEGLOTICASE RESPONSE RATE IN UNCONTROLLED GOUT PATIENTS CO-TREATED WITH METHOTREXATE: EXPERIENCE IN A COMMUNITY RHEUMATOLOGY PRACTICE}

J. Albert ${ }^{1}$, T. Hosey ${ }^{2}$, B. Lamoreaux ${ }^{2} .{ }^{1}$ Rheumatic Disease Center, Glendale, United States of America; ${ }^{2}$ Horizon Therapeutics, Lake Forest, United States of America

Background: Pegloticase is an infused biologic approved to treat uncontrolled gout. The drug is highly effective, but patients can develop anti-drug antibodies that interfere with efficacy. ${ }^{1}$ Randomized clinical trials have shown that $42 \%$ of patients treated with bi-weekly pegloticase had a serum uric acid (sUA) below $6.0 \mathrm{mg} / \mathrm{dl}$ at 3 and 6 months. $^{2}$ Mild-to-moderate immunomodulation has been shown to lower the prevalence of anti-drug antibody formation in patients with other autoimmune diseases (rheumatoid arthritis, Crohn's disease, juvenile idiopathic arthritis). ${ }^{3}$ Cases published in the literature suggest that low-to-moderate doses of methotrexate ${ }^{4,5}$ or azathioprine ${ }^{6}$ may also attenuate anti-pegloticase antibody formation in uncontrolled gout patients. Therefore, immunomodulation may allow patients to remain on pegloticase therapy longer and achieve a more complete therapeutic response.

Objectives: To examine pegloticase treatment response in patients co-treated with methotrexate.

Methods: This retrospective chart review included patients from a single community rheumatology practice who began pegloticase ( $8 \mathrm{mg}$ every 2 weeks) therapy between January 2017 and September 2019 and were co-treated with methotrexate. Unless contraindicated, methotrexate co-treatment with pegloticase is now standard in this practice and all patients undergo close monitoring of laboratory parameters including serum uric acid level (sUA), blood counts, and liver function tests (LFTs). To maximize the number of cases, patients administered methotrexate in any form were included. Collected data included demographic information, laboratory values, methotrexate treatment parameters (timing with respect to pegloticase therapy, dose, route), pegloticase response parameters (number of infusions, duration of therapy), and adverse events. Main outcome measures included the number of pegloticase infusions administered (responder defined as $\geq 12$ infusions administered) and therapy duration.

Results: Ten patients ( 9 male) were included. All patients had visible tophi and average patient age was $52.3 \pm 13.5$ years. Nine patients began subcutaneous methotrexate ( $25 \mathrm{mg}$ weekly) an average of $19.9 \pm 7.0$ days (range: 14 to 35 days) before the first pegloticase infusion. The remaining patient began oral methotrexate (12.5 mg weekly) 14 days after the first pegloticase infusion. Eight of 10 patients ( $80 \%$ ) were considered responders, receiving an average of $15.5 \pm$ 3.8 pegloticase infusions (range: $12-21$ infusions) over $31.8 \pm 9.5$ weeks (range: 22.1 to 48.3 weeks). In these 8 responders, mean sUA was $0.2 \pm 0.0 \mathrm{mg} / \mathrm{dL}$ immediately prior to the last pegloticase infusion. All 10 patients had an initial, rapid decrease in SUA, but two patients discontinued treatment before infusion 12. One patient had increased sUA with a mild infusion reaction, and one patient was lost to follow-up after infusion 5 . No new safety concerns emerged. A gout flare occurred in 1 patient and was treated with prednisone. LFT and blood cell parameters were stable over the study period, except in two patients. One had a mild, transient LFT elevation that resolved without treatment, one had an LFT elevation and pancytopenia that improved with methotrexate discontinuation and transfusion, respectively. This patient remained on pegloticase and continued as a responder.

Conclusion: This case series suggests that methotrexate, when used as a co-therapy with pegloticase, allows more patients to complete therapy and to achieve the full therapeutic response. No new safety concerns emerged.

References:

[1] Lipsky PE, et al. Arthritis Res Ther 2014;16:R60.

[2] Sundy JS, et al. JAMA 2011;306:711-20.

[3] Krieckaert CL, et al. Arthritis Res Ther 2010;12:217. 